\title{
DIFFUSION UNDER GEOMETRICAL CONSTRAINT
}

\section{NAOHISA OGAWA}

Communicated by Ivaïlo M. Mladenov

\begin{abstract}
Here we discus the diffusion of particles in a curved tube. This kind of transport phenomenon is observed in biological cells and porous media. To solve such a problem, we discuss the three dimensional diffusion equation with a confining wall forming a thinner tube. We find that the curvature appears in a effective diffusion coefficient for such a quasi-one-dimensional system. As an application to higher dimensional case, we discuss the diffusion in a curved surface with thickness. In this case the diffusion coefficient changes to the tensor form depending on the mean and Gaussian curvatures. Then the diffusion flow can be interpreted as usual flow plus anomalous flow. The anomalous flow shows not only the diffusion but also the concentration depending on mean and Gaussian curvatures, and also it includes the flow proportional to the gradient of Gaussian curvature.
\end{abstract}

\section{Introduction}

The particle motion on a given curved manifold (surface) is old but interesting problem. It shows not only the check of general relativity, but also the physical phenomena in a scale around us. But in latter case, precisely saying, it is the curved line with girth $\left(M_{1} \times \mathbb{R}^{2}\right)$ and the curved surface with thickness $\left(M_{2} \times \mathbb{R}\right)$ embedded in the three-dimensional Euclidean space $\mathbb{R}^{3}$. Usually we neglect the effect of the girth and the thickness, and we identify the system as the motion on a curved line and curved surface. This is true when the length parameters $\left\{l_{1}, l_{2}, \cdots l_{N}\right\}$ in $M$ and thickness $\epsilon$ of the system satisfies the conditions

$$
l_{i}>>\epsilon, \quad i=1,2, \ldots N
$$

where $l_{i}$ are the parameters with dimension of the length in $M$. One such example is $1 / \kappa$, where $\kappa$ is the local curvature of $M$. But when the thickness (or grith) $\epsilon$ is comparable to the inverse of curvature, it is no longer true even though all of other length parameters are satisfying the condition (1). In such a case, we can construct the effective equation in one or two dimensional manifolds by taking into account the effect of the girth and thickness. 
In this paper we consider first the diffusion equation in a curved tube: $M_{1} \times \mathbb{R}^{2}$ with circular cross section which radius equals to $\epsilon$, and construct the effective one-dimensional diffusion equation. Then we show that the effect of the girth is appearing in diffusion coefficient as a function of $\epsilon \kappa$ [17]. Next we discuss the higher dimensional case, i.e., curved surface with thickness: $M_{2} \times \mathbb{R}$. The thickness effect is important when the curvature radius is similar in size to the thickness as before. We introduce a diffusion equation in the three-dimensional space, then confine the particles on the two-dimensional curved surface plus thickness, and finally consider how the diffusion equation is modified. The obtained diffusion equation has diffusion coefficient that depends on a tensor formed by both of mean and Gauss curvature. And surprisingly we have a new diffusion flow that is driven by a gradient of the Gaussian curvature [16]. The mathematical tool used here is similar to the one for the quantum mechanics on curved spaces $[4,7,15]$.

This kind of analysis is important to control the transportation of micro- and nanoparticles artificially. And such phenomena are encountered in biological cells [1] zeolite [13], and in catalytic reactions in porous media [5]. For those purposes, the diffusion properties in confined geometries are discussed by several authors. The diffusion in a membrane with a certain thickness is discussed by Gov [9], Gambin et al [8] and Ogawa [16]. The diffusion in general curved manifold is discussed by Castro-Villarreal [3]. The diffusion in a tube with a varying cross section along the axis (channel model) is discussed by Jacobs [11], Yanagida [19], Zwanzig [20], Reguera and Rubi [18], Kalinary and Percus [12], and reviewed, by Burada et al and Hänggi $[2,10]$.

In Section 2, we introduce the curvilinear coordinates and related metrics in a winding tube. This is carried out by using Frenet-Serret (FS) equations. In Section 3, we define the quasi one-dimensional diffusion field and the diffusion equation is obtained by using a local equilibrium condition. In Section 4, we calculate the mean square displacement (MSD) from the quasi one-dimensional diffusion equation and find the first two terms in the short time expansion by using curvature and its derivatives. In Section 5, the extension to the curved surface with thickness is given. In Section 6, properties of the anomalous diffusion flow is explained. Section 7 presents the conclusion.

\section{Geometry of a Curved Tube}

We set the curved tube with a radius $\epsilon$ in the three-dimensional Euclidean space $\mathbb{R}^{3}$. The curvilinear coordinate which we utilize are as follows. 
The vector $\vec{X}$ presents the Cartesian coordinates in $\mathbb{R}^{3}, s\left(=q^{1}\right)$ is the arclength parameter along the center line of the tube and $\vec{e}_{1}$ is its tangential vector. The vector $\vec{x}(s)$ specifies the Cartesian coordinates of the points on the center line and $q^{i}$ are the coordinates in the transversal directions $\vec{e}_{i}$. The small Latin indices $i, j, k, \cdots$ run from 2 to 3 and the Greek indices $\mu, \nu, \cdots$ run from 1 to 3 . Sometimes we use the notation $s=q^{1}, v=q^{2}=r \cos \theta$, and $w=q^{3}=r \sin \theta$ to obtain simpler expressions, and we define the area element of the cross section as $\mathrm{d} A=\mathrm{d} v \mathrm{~d} w=$ $r \mathrm{~d} r \mathrm{~d} \theta$. The vectors $\vec{e}_{1}, \vec{e}_{2}$, and $\vec{e}_{3}$ are the unit basis vectors introduced by the FS equations with two geometrical quantities $\kappa$-curvature and the torsion $\tau$

$$
\begin{aligned}
\frac{\mathrm{d} \vec{e}_{1}}{\mathrm{~d} s} & =\kappa \vec{e}_{2} \\
\frac{\mathrm{d} \overrightarrow{e_{2}}}{\mathrm{~d} s} & =-\kappa \vec{e}_{1}+\tau \vec{e}_{3} \\
\frac{\mathrm{d} \vec{e}_{3}}{\mathrm{~d} s} & =-\tau \vec{e}_{2} .
\end{aligned}
$$

Then, we identify any point in the tube using the parameterization described above via the formula

$$
\vec{X}\left(s, q^{2}, q^{3}\right)=\vec{x}(s)+q^{i} \vec{e}_{i}(s), \quad q^{i} \in \mathbb{R}, \quad i=2,3
$$

where $0 \leq|\vec{q}| \leq \epsilon$ with $|\vec{q}|=\sqrt{\left(q^{2}\right)^{2}+\left(q^{3}\right)^{2}}$.

Then we obtain the curvilinear coordinate system in the tube $\left(\subset \mathbb{R}^{3}\right)$ using the coordinates $q^{\mu}=\left(q^{1}, q^{2}, q^{3}\right)$ and the metric $G_{\mu \nu}$

$$
G_{\mu \nu}=\frac{\partial \vec{X}}{\partial q^{\mu}} \cdot \frac{\partial \vec{X}}{\partial q^{\nu}} .
$$

The metric tensor $G_{\mu \nu}$ is calculated by using the Frenet - Serret equations which produce

$$
G_{\mu \nu}=\left(\begin{array}{ccc}
1-2 \kappa v+\left(\kappa^{2}+\tau^{2}\right) v^{2}+\tau^{2} w^{2} & -\tau w & \tau v \\
-\tau w & 1 & 0 \\
\tau v & 0 & 1
\end{array}\right)
$$

The determinant of the metric tensor is given by the formula

$$
G \equiv \operatorname{det}\left(G_{\mu \nu}\right)=(1-\kappa v)^{2}
$$

and the inverse metric is

$$
G^{\mu \nu}=\frac{1}{(1-\kappa v)^{2}}\left(\begin{array}{ccc}
1 & \tau w & -\tau v \\
\tau w & (1-\kappa v)^{2}+(\tau w)^{2} & -\tau^{2} v w \\
-\tau v & -\tau^{2} v w & (1-\kappa v)^{2}+(\tau v)^{2}
\end{array}\right) .
$$




\section{Quasi One-Dimensional Diffusion Equation}

Let us define a three-dimensional diffusion field by $\phi^{(3)}$ and a three-dimensional Laplace-Beltrami operator associated with the metric tensor (7) by $\hat{\Delta}$. Then we have the diffusion equation

$$
\frac{\partial \phi^{(3)}}{\partial t}=D \hat{\Delta} \phi^{(3)}
$$

where $D$ is the diffusion constant. Our aim is to construct the effective onedimensional diffusion equation from the $3 \mathrm{D}$ equation above in a small radius limit

$$
\frac{\partial \phi^{(1)}}{\partial t}=D \hat{\Delta}^{(\mathrm{eff})} \phi^{(1)}
$$

where $\phi^{(1)}$ is the one-dimensional diffusion field and $\hat{\Delta}^{(\text {eff })}$ is the unknown effective 1D diffusion operator. The particles number in $s \sim s+\mathrm{d} s$ should be the same, i.e.,

$$
\mathrm{d} s \int \phi^{(3)}\left(s, q^{2}, q^{3}\right) \sqrt{G} \mathrm{~d} A=\phi^{(1)}(s) \mathrm{d} s .
$$

From the above condition, we obtain

$$
\phi^{(1)}(s)=\int \phi^{(3)} \sqrt{G} \mathrm{~d} A .
$$

We multiply equation (10) by $\sqrt{G}$ and integrate it with respect to $A$ in order to obtain

$$
\frac{\partial \phi^{(1)}}{\partial t}=D \int(\sqrt{G} \hat{\Delta}) \phi^{(3)} \mathrm{d} A .
$$

From the expression of the Laplace-Beltrami operator

$$
\Delta=G^{-1 / 2} \frac{\partial}{\partial q^{\mu}} G^{1 / 2} G^{\mu \nu} \frac{\partial}{\partial q^{\nu}}
$$

our diffusion equation takes the form

$$
\frac{\partial \phi^{(1)}}{\partial t}=D \int \frac{\partial}{\partial q^{\mu}} G^{1 / 2} G^{\mu \nu} \frac{\partial}{\partial q^{\nu}} \phi^{(3)} \mathrm{d} A=D \frac{\partial}{\partial s} \int \frac{1}{\sqrt{G}}\left(\frac{\partial}{\partial s}-\tau \frac{\partial}{\partial \theta}\right) \phi^{(3)} \mathrm{d} A
$$

where the Neumann boundary condition is used for the second equality.

The fluctuation mode in the cross section decreases with time like $\exp \left(-D t / \epsilon^{2}\right)$, and only the zero mode (uniform in the same cross section) survives at $t>>\epsilon^{2} / D$, 
i.e., the equilibrium is realized in the transverse direction in a short time. Then we suppose the validity of the "local equilibrium conditions" as

$$
\frac{\partial \phi^{(3)}}{\partial q^{i}}=0, \quad i=2,3
$$

Whether these conditions can be used or not when the curvature is large is not yet clear, however, we can prove the result without using local equilibrium conditions by the help of Markov approximation, though it is much complicated [17]. In the following we restrict our considerations in the time scale $t$ satisfying $t>>\epsilon^{2} / D$ and we assume the local equilibrium conditions (16). From equations (13) and (16), we also obtain

$$
\phi^{(3)}=\frac{\phi^{(1)}}{\sigma}, \quad \sigma \equiv \int \sqrt{G} \mathrm{~d} A=\pi \epsilon^{2} .
$$

From (15) and (17), we obtain

$$
\frac{\partial \phi^{(1)}}{\partial t}=\frac{\partial}{\partial s} D_{\mathrm{eff}} \frac{\partial \phi^{(1)}}{\partial s}
$$

where

$$
\begin{aligned}
D_{\text {eff }} & \equiv \frac{D}{\pi \epsilon^{2}} \int \frac{\mathrm{d} A}{\sqrt{G}}=D\left(1+\frac{1}{4}(\kappa \epsilon)^{2}+\frac{1}{8}(\kappa \epsilon)^{4}+\frac{5}{64}(\kappa \epsilon)^{6}+\cdots\right) \\
& =2 D \frac{1-\sqrt{1-(\kappa \epsilon)^{2}}}{(\kappa \epsilon)^{2}}, \quad|\kappa \epsilon|<1 .
\end{aligned}
$$

Actually we have the representation

$$
D_{\text {eff }}=D\left\langle\frac{1}{1-\kappa q^{2}}\right\rangle, \quad\langle\ldots\rangle=\frac{1}{\pi \epsilon^{2}} \int \mathrm{d} A \ldots
$$

Then, we find a simple interpretation of the effective diffusion coefficient. Let us consider the point $P$ on the tube where the curvature is $\kappa$. We select two sections around $P$ and discuss the length connecting these two sections (see Fig. 1). At the coordinate $q^{2}$, the length between the two sections is given by $s$, which is different from the length of the center line, $\bar{s}$, with the ratio

$$
\frac{\bar{s}}{s}=\frac{1}{1-\kappa q^{2}} .
$$

Next, we should note that the relation between diffusion flow and density difference is similar to Ohm's law

$$
J \sigma=\frac{D \sigma}{s} \Delta N \sim I=\frac{1}{R} V
$$




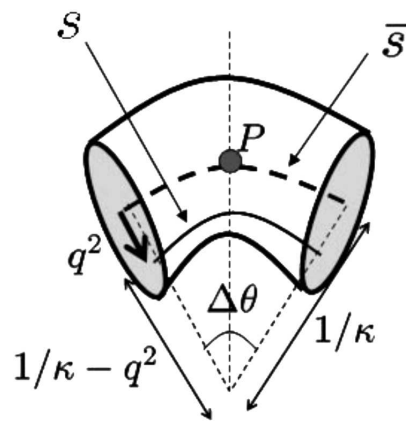

Figure 1. Bent point $P$ of tube: $q^{2}$ shows distance to the direction of center of curvature. The center line has the curvature radius $1 / \kappa$ and the line with $q^{2} \neq 0$ has the curvature radius $1 / \kappa-q^{2}$.

where $J$ is the flow density, $\sigma$ is the cross section, $D$ is the diffusion constant, $s$ is the distance, and $\Delta N$ is the density difference. In the comparison with Ohm's law, electric current corresponds to $J \sigma$, voltage corresponds to $\Delta N$, and electric conductivity $1 / R$ corresponds to $D \sigma / s$. By using the above correspondence, we consider our tube as a bundle of thin tubes (see Fig. 2). Thus, it can be seen as a parallel connection of many resistances. The total conductivity is calculated as

$$
\frac{1}{R}=\sum_{i=1}^{N} \frac{1}{R_{i}}=D \sum_{i=1}^{N} \frac{\Delta A_{i}}{s_{i}} \equiv D_{\text {eff }} \frac{\sigma}{\bar{s}}
$$

where $\sigma=\sum_{i} \Delta A_{i}$. The last equality is just the definition of the effective diffusion coefficient. Thus, we obtain

$$
D_{\text {eff }}=\frac{D}{\sigma} \sum_{j=1}^{N} \frac{\bar{s}}{s_{j}} \Delta A_{j}=\frac{D}{\pi \epsilon^{2}} \int \frac{\mathrm{d} A}{1-\kappa q^{2}}=D\left\langle\frac{1}{1-\kappa q^{2}}\right\rangle
$$

where (21) is utilized at the second equality.

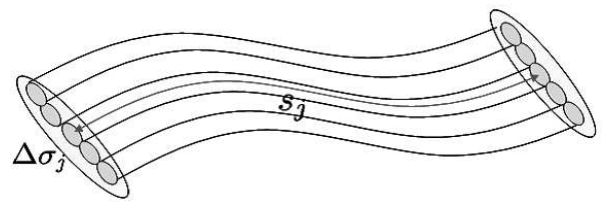

Figure 2. Bundle of tubes: our tube is considered as a bundle of infinitesimally thin tubes with each length $s_{j}$ and cross-sectional area $\Delta A_{j}$.

The physical reason why the diffusion coefficient increases at the curved point is similar to the discussion of total resistance for the following electric circuit. Let us 
consider the parallel connection of three electric resistances. When the resistances have the same $R$ value, the total resistance is $R / 3$. However, when the resistances have a dispersion $r$ with a mean $R$ value $(r<<R)$, such as $R_{1}=R+r, R_{2}=R$, and $R_{3}=R-r$, the total resistance takes the value

$$
R_{\mathrm{tot}}=\frac{R}{3}\left[1-\frac{2}{3}\left(\frac{r}{R}\right)^{2}\right]
$$

The dispersion reduces the total resistance, i.e., increases the diffusion coefficient, as is shown in (19).

\section{Mean Square Displacement}

Our quasi one-dimensional diffusion equation (18) determines the time development of MSD as follows. From the definition of the expectational value, we have

$$
\langle f(s)\rangle \equiv \frac{\int f(s) \phi(s, t) \mathrm{d} s}{\int \phi(s, t) \mathrm{d} s} .
$$

Then, we obtain

$$
\frac{\partial}{\partial t}\left\langle(\Delta s)^{2}\right\rangle=2\left\langle D_{\text {eff }}(s)\right\rangle+2\left\langle(\Delta s) D_{\text {eff }}^{\prime}(s)\right\rangle
$$

and

$$
\begin{aligned}
\frac{\partial^{2}}{\partial t^{2}}\left\langle(\Delta s)^{2}\right\rangle= & 6\left\langle D_{\mathrm{eff}}^{\prime \prime}(s) D_{\mathrm{eff}}(s)\right\rangle+2\left\langle D_{\mathrm{eff}}^{\prime}(s)^{2}\right\rangle \\
& +2\left\langle D_{\mathrm{eff}}^{\prime \prime \prime}(s) D_{\mathrm{eff}}(s)(\Delta s)\right\rangle+2\left\langle D_{\mathrm{eff}}^{\prime \prime}(s) D_{\mathrm{eff}}^{\prime}(s)(\Delta s)\right\rangle
\end{aligned}
$$

where $\Delta s \equiv s-\langle s\rangle$.

Let us choose the initial condition as follows

$$
\phi(t=0, s)=\delta(s)
$$

Then, we calculate MSD by short time expansion near $t \sim 0$

$$
\left\langle(\Delta s)^{2}\right\rangle=a_{1} t+a_{2} t^{2}+\ldots
$$

so that

$$
a_{1}=\frac{\partial\left\langle(\Delta s)^{2}\right\rangle}{\partial t}{ }_{t=0}=2 D\left(1+\frac{1}{4}(\kappa \epsilon)^{2}+\frac{1}{8}(\kappa \epsilon)^{4}+\frac{5}{64}(\kappa \epsilon)^{6}+\ldots\right)_{s=0}
$$




$$
\begin{aligned}
a_{2}= & \frac{1}{2} \frac{\partial^{2}\left\langle(\Delta s)^{2}\right\rangle}{\partial t^{2}} t_{t=0} \\
= & D^{2} \epsilon^{2}\left(\kappa^{\prime \prime} \kappa\right)\left(\frac{3}{2}+\frac{15}{8}(\kappa \epsilon)^{2}+\frac{63}{32}(\kappa \epsilon)^{4}+\cdots\right)_{s=0} \\
& +D^{2} \epsilon^{2} \kappa^{\prime 2}\left(\frac{3}{2}+\frac{41}{8}(\kappa \epsilon)^{2}+\frac{283}{32}(\kappa \epsilon)^{4}+\cdots\right)_{s=0}
\end{aligned}
$$

and where we have made use of equation (19).

Note that these short time expansion coefficients are given by the curvature and its space derivatives. This procedure is similar to that given in a theory of diffusion in higher dimensional Riemannian manifold [3], although the coefficients are determined by the Riemannian curvature that vanishes in our case. In this way, we obtain the curvature dependent MSD for the quasi-one-dimensional curved system.

\section{Extension to the Curved Surface with Thickness}

Next we consider a curved surface $\Sigma$ embedded in three dimensional Euclidean space $\mathbb{R}^{3}$, and we put two similar copies of both sides of $\Sigma$, called $\tilde{\Sigma}$ and $\Sigma^{\prime}$ at a small distance $\epsilon / 2$. Our particles can only move between these two surfaces, and later we take the limit $\epsilon \rightarrow 0$. We look for the form of the diffusion equation in this limit. The coordinates we use hereafter are the following (see Fig. 3).

As before $\vec{X}$ is the Cartesian coordinate in $\mathbb{R}^{3}$ and $\vec{x}$ is the Cartesian coordinate which specifies only the points on $\Sigma$ and $q^{i}$ is the curved coordinate on $\Sigma$ and $q^{0}$ is the coordinate in $\mathbb{R}^{3}$ normal to $\Sigma$. Further by using the normal unit vector $\vec{n}\left(q^{1}, q^{2}\right)$ on $\Sigma$ at point $\left(q^{1}, q^{2}\right)$, we can identify any point between two surfaces $\Sigma^{\prime}$ and $\tilde{\Sigma}$ by the following thin-layer approximation [7]

$$
\vec{X}\left(q^{0}, q^{1}, q^{2}\right)=\vec{x}\left(q^{1}, q^{2}\right)+q^{0} \vec{n}\left(q^{1}, q^{2}\right)
$$

where $-\epsilon / 2 \leq q^{0} \leq \epsilon / 2$.

From this relation we can obtain the curvilinear coordinate system between two surfaces $\left(\subset \mathbb{R}^{3}\right)$ with coordinates $q^{\mu}=\left(q^{0}, q^{1}, q^{2}\right)$, and metric $G_{\mu \nu}$

$$
G_{\mu \nu}=\frac{\partial \vec{X}}{\partial q^{\mu}} \cdot \frac{\partial \vec{X}}{\partial q^{\nu}}, \quad \mu, v=0,1,2 .
$$

The different blocks $G_{\mu \nu}$ are as follows

$$
G_{i j}=g_{i j}+q^{0}\left(\frac{\partial \vec{x}}{\partial q^{i}} \cdot \frac{\partial \vec{n}}{\partial q^{j}}+\frac{\partial \vec{x}}{\partial q^{j}} \cdot \frac{\partial \vec{n}}{\partial q^{i}}\right)+\left(q^{0}\right)^{2} \frac{\partial \vec{n}}{\partial q^{i}} \cdot \frac{\partial \vec{n}}{\partial q^{j}}
$$




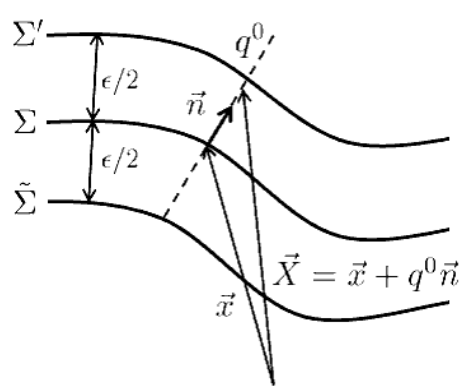

Figure 3. Embedding and coordinates.

where

$$
g_{i j}=\frac{\partial \vec{x}}{\partial q^{i}} \cdot \frac{\partial \vec{x}}{\partial q^{j}}
$$

is the metric on $\Sigma$. Hereafter the indices $i, j, k \cdots$ are lowered or rised by $g_{i j}$ and its inverse $g^{i j}$. We also obtain

$$
G_{0 i}=G_{i 0}=0, \quad G_{00}=1 .
$$

Now we can proceed with the calculations by using the new variables. We first define the tangential vector to $\Sigma$ as

$$
\vec{B}_{k}=\frac{\partial \vec{x}}{\partial q^{k}}
$$

Note that $\vec{n} \cdot \vec{B}_{k}=0$. Then we obtain two kinds of relations.

The Gauss equations

$$
\frac{\partial \vec{B}_{i}}{\partial q^{j}}=-\kappa_{i j} \vec{n}+\Gamma_{i j}^{k} \vec{B}_{k}
$$

where the Christoffel symbols are defined by the equalities

$$
\Gamma_{i j}^{k} \equiv \frac{1}{2} g^{k m}\left(\partial_{i} g_{m j}+\partial_{j} g_{i m}-\partial_{m} g_{i j}\right)
$$

and the Weingarten equations

$$
\frac{\partial \vec{n}}{\partial q^{j}}=\kappa_{j}^{m} \vec{B}_{m}
$$

in which $\kappa_{i j}$ are the components of the so called Euler-Schouten tensor, or second fundamental tensor defined by the formula

$$
\kappa_{i j}=\frac{\partial \vec{n}}{\partial q^{i}} \cdot \vec{B}_{j} .
$$


The second fundamental tensor $\kappa_{i j}$ is the projection of the vector $\partial \vec{n}$ onto the surface. Furthermore, the mean curvature is given by

$$
\kappa=g^{i j} \kappa_{i j}
$$

and the Ricci scalar curvature $R$ is defined as

$$
R / 2=\operatorname{det}\left(\kappa_{j}^{i}\right)=\frac{1}{2}\left(\kappa^{2}-\kappa_{i j} \kappa^{i j}\right) .
$$

In this way we have the formula for metric of curvilinear coordinate in a neighborhood of $\Sigma$, i.e.,

$$
G_{i j}=g_{i j}+2 q^{0} \kappa_{i j}+\left(q^{0}\right)^{2} \kappa_{i m} \kappa_{j}^{m} .
$$

Now we have also the total metric tensor

$$
G_{\mu \nu}=\left(\begin{array}{cc}
1 & 0 \\
0 & G_{i j}
\end{array}\right)
$$

Let us denote the three-dimensional diffusion field as $\phi^{(3)}$, and the three-dimensional Laplacian as $\Delta^{(3)}$. Then we have the equation

$$
\frac{\partial \phi^{(3)}}{\partial t}=D \Delta^{(3)} \phi^{(3)}
$$

where $D$ is the diffusion constant, $G=\operatorname{det}\left(G_{\mu \nu}\right)=\operatorname{det}\left(G_{i j}\right)$. Our aim is to construct the effective two dimensional diffusion equation from the $3 \mathrm{D}$ equation above, namely

$$
\frac{\partial \phi^{(2)}}{\partial t}=D \Delta^{(\mathrm{eff})} \phi^{(2)}
$$

where $\phi^{(2)}$ is the two dimensional diffusion field, $g=\operatorname{det}\left(g_{i j}\right)$, and $\Delta^{(\text {eff })}$ is the unknown effective 2D diffusion operator which might not be equal to the $2 \mathrm{D}$ Laplace-Beltrami operator. The particle number in $\mathrm{d}^{2} q=\mathrm{d} q^{1} \mathrm{~d} q^{2}$ should be the same

$$
\phi^{(2)}\left(q^{1}, q^{2}\right) \sqrt{g} \mathrm{~d}^{2} q=\mathrm{d}^{2} q \int \phi^{(3)}\left(q^{0}, q^{1}, q^{2}\right) \sqrt{G} \mathrm{~d} q^{0} .
$$

From the above condition, we obtain

$$
\phi^{(2)}\left(q^{1}, q^{2}\right)=\int_{-\epsilon / 2}^{\epsilon / 2} \tilde{\phi}^{(3)} \mathrm{d} q^{0}
$$

where

$$
\tilde{\phi}^{(3)} \equiv \phi^{(3)} \sqrt{G / g} .
$$


By multiplying the equation (44) with $\sqrt{G / g}$ and integration with respect to $q^{0}$, we obtain

$$
\frac{\partial \phi^{(2)}}{\partial t}=D \int_{-\epsilon / 2}^{\epsilon / 2} \tilde{\Delta}^{(3)} \tilde{\phi}^{(3)} \mathrm{d} q^{0}
$$

where

$$
\tilde{\Delta}^{(3)} \equiv \sqrt{G / g} \Delta^{(3)} \sqrt{g / G}
$$

Thus our two-dimensional effective diffusion equation up to $O\left(\epsilon^{2}\right)$ terms is

$$
\begin{aligned}
\frac{\partial \phi^{(2)}}{\partial t}= & D \Delta^{(2)} \phi^{(2)}+D \hat{A} \int_{-\epsilon / 2}^{\epsilon / 2} q^{0} \tilde{\phi}^{(3)} \mathrm{d} q^{0} \\
& +D \hat{B} \int_{-\epsilon / 2}^{\epsilon / 2}\left(q^{0}\right)^{2} \tilde{\phi}^{(3)} \mathrm{d} q^{0}+O\left(\epsilon^{3}\right)
\end{aligned}
$$

where

$$
\begin{gathered}
\hat{A}=-g^{-1 / 2} \frac{\partial}{\partial q^{i}} g^{1 / 2}\left(2 \kappa^{i j} \frac{\partial}{\partial q^{j}}+g^{i j} \frac{\partial \kappa}{\partial q^{j}}\right) \\
\hat{B}=g^{-1 / 2} \frac{\partial}{\partial q^{i}} g^{1 / 2}\left(3 \kappa^{i m} \kappa_{m}^{j} \frac{\partial}{\partial q^{j}}+\frac{1}{2} g^{i j} \frac{\partial\left(\kappa^{2}-R\right)}{\partial q^{j}}+2 \kappa^{i j} \frac{\partial \kappa}{\partial q^{j}}\right) .
\end{gathered}
$$

To proceed with the $q^{0}$ integration, we suppose that there is no diffusion flow in the normal direction to the layer, that is

$$
0=\frac{\partial \phi^{(3)}}{\partial q^{0}}=g^{1 / 2} \frac{\partial G^{-1 / 2} \tilde{\phi}^{(3)}}{\partial q^{0}}
$$

In this case the solution is

$$
\tilde{\phi}^{(3)}=\frac{1}{K}(G / g)^{1 / 2} \phi^{(2)}\left(q^{1}, q^{2}\right), \quad K \equiv \int_{-\epsilon / 2}^{\epsilon / 2}(G / g)^{1 / 2} \mathrm{~d} q^{0} .
$$

The integrations in (51) can be performed explicitly, and we obtain the final form of the equation up to $O\left(\epsilon^{2}\right)$ terms as

$$
\begin{aligned}
\frac{\partial \phi^{(2)}}{\partial t}= & D \Delta^{(2)} \phi^{(2)}+\tilde{D} g^{-1 / 2} \frac{\partial}{\partial q^{i}} g^{1 / 2} \\
& \times\left(\left(3 \kappa^{i m} \kappa_{m}^{j}-2 \kappa \kappa^{i j}\right) \frac{\partial}{\partial q^{j}}-\frac{1}{2} g^{i j} \frac{\partial R}{\partial q^{j}}\right) \phi^{(2)}
\end{aligned}
$$

where $\tilde{D}=\frac{\epsilon^{2}}{12} D$ 
The normal diffusion flow can be written in general as

$$
J_{N}^{i}=-D g^{i j} \frac{\partial \phi^{(2)}}{\partial q^{j}}
$$

and the anomalous diffusion flow is

$$
J_{A}^{i}=-\tilde{D}\left(\left(3 \kappa^{i m} \kappa_{m}^{j}-2 \kappa \kappa^{i j}\right) \frac{\partial \phi^{(2)}}{\partial q^{j}}-\frac{1}{2} g^{i j} \frac{\partial R}{\partial q^{j}} \phi^{(2)}\right) .
$$

Finally, the diffusion equation can be written as

$$
-\frac{\partial \phi^{(2)}}{\partial t}=\nabla_{i}\left(J_{N}^{i}+J_{A}^{i}\right)
$$

in which $\nabla_{i}$ are the covariant derivatives.

\section{Anomalous Diffusion Flow: Diffusion and Concentration}

The anomalous flow is equal to zero for the flat surface. The last term in equation (58) shows that curvature gradient generate the flow without gradient of particle number density. From the signature of this term, this flow goes from the smaller Ricci scalar point to the larger Ricci scalar point. (Ricci scalar $R$ is related to Gauss curvature by the equality $R / 2=\operatorname{det}\left[\kappa_{j}^{i}\right]$.) To consider the first term let us work with the coordinates which satisfy

$$
g_{i j}=\delta_{i j}, \quad \kappa_{j}^{i}=\operatorname{diag}\left[1 / r_{1}, 1 / r_{2}\right]
$$

at the point $P$, where $r_{i}$ are the curvature radius along the $q^{i}$ coordinate and it takes positive or negative value for convex or concave. The metric can be diagonalized by choosing the two coordinates to be orthogonal, and it can be normalized by using the re-parametrization. The second fundamental tensor is diagonalized by rotation of the coordinate system.

Then we have positive or negative value for

$$
f^{i j} \equiv 3 \kappa^{i m} \kappa_{m}^{j}-2 \kappa \kappa^{i j}
$$

depending on the value of the curvature. In our coordinates, we can immediately write it in the simple form

$$
f^{i j}=\delta^{i j}\left(\frac{1}{r_{i}^{2}}-\frac{2}{r_{1} r_{2}}\right) .
$$




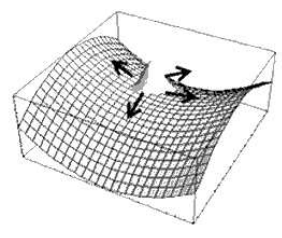

a

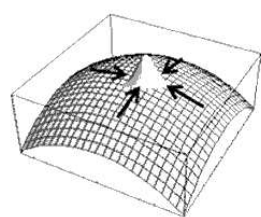

b

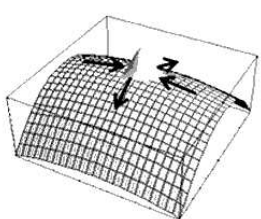

c

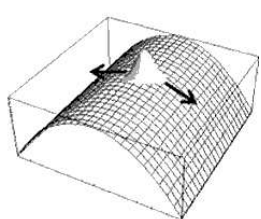

d

Figure 4. Wave packet on curved surface.

When the surface is hyperbolic $\left(R<0 ; r_{1} r_{2}<0\right), \quad f^{11}>0, f^{22}>0$ and the usual diffusion occurs (see Fig. 4 a).

When the surface is convex or concave $\left(R>0 ; r_{1} r_{2}>0\right)$, we have three possibilities.

One possibility is that $f^{11}<0, f^{22}<0$, when

$$
1 / 2<\left|\frac{r_{2}}{r_{1}}\right|<2
$$

Then we have no diffusion but concentration occurs (see Fig. 4 b).

The second possibility is that $f^{11} f^{22}<0$, if

$$
\left|\frac{r_{2}}{r_{1}}\right|<1 / 2 \quad \text { or } \quad\left|\frac{r_{2}}{r_{1}}\right|>2 \text {. }
$$

Then we have diffusion in one direction, but concentration in another direction (see Fig. 4 c).

We have also a critical point between above two regions: when $f^{11} f^{22}=0$, so that

$$
\left|\frac{r_{2}}{r_{1}}\right|=1 / 2 \quad \text { or } \quad\left|\frac{r_{2}}{r_{1}}\right|=2 .
$$

But such a point arises as a discrete point or as continuous line, and do not extend on surface.

When the Ricci scalar is zero $(R=0)$, for example $r_{2}=\infty, f^{22}=0$ and $f^{11}>0$, the diffusion occurs only in $q^{1}$ direction but not in another direction (see Fig. $4 \mathrm{~d}$ ). In this way, this anomalous diffusion flow has much varieties depending on the curvature.

\section{Conclusion}

We have presented two examples of diffusion under geometrical constraint. The geometrical constraint reduces the three dimensional diffusion equation to the lower 
dimensional effective equation depending on the curvature of constraint condition. In the case of a tube, the curvature dependent diffusion coefficient appears, and in the case of a curved surface with thickness, the anomalous diffusion flow appears and not only the diffusion but also the concentration flow appears related to the form of the second fundamental tensor. Further additional flow proportional to the gradient of Gaussian curvature is also obtained.

\section{References}

[1] Alberts B., Johnson A., Lewis J., Raff M., Roberts K. and Walter P., Molecular Biology of the Cell, Garland, New York 2007.

[2] Burada P., Hänggi P., Marhesoni F., Schmid G. and Talkner P., Diffusion in Confined Geometries, Chem. Phys. Chem. 10 (2009) 45-54 (see also the references therein).

[3] Castro-Villarreal P., Brownian Motion Meets Riemann Curvature, J. Stat. Mech. (2010) P08006 (16pp).

[4] da Costa R., Quantum Mechanics of a Constrained Particle, Phys. Rev. A 23 (1981) 1982-1987; Tolar J., On a Quantum Mechanical d'Alembert Principle, Lecture Notes in Physics 313 (1988) 268-274.

[5] Daniel M. and Astruc D., Gold Nanoparticles: Assembly, Supramolecular Chemistry, Quantum-Size-Related Properties, and Applications Toward Biology, Catalysis, and Nanotechnology, Chem. Rev. 104 (2004) 293-346.

[6] Faraudo J., Diffusion Equation on Curved Surfaces. I. Theory and Application to Biological Membranes, J. Chem. Phys 116 (2002) 5831-5841; Balakrishnan J., Spatial Curvature Effects on Molecular Transport by Diffusion, Phys. Rev. E 61 (2000) 4648-4651.

[7] Fujii K. and Ogawa N., Generalization of Geometry-Induced Gauge Structure to Any Dimensional Manifold, Progr. Theor. Phys. 89 (1993) 575-578.

[8] Gambin Y., Esparza R., Reffay M., Sierecki E., Gov N., Genest M., Hodges R. and Urbach W., Lateral Mobility of Proteins in Liquid Membranes Revisited, Proc. Natl. Acad. Sci. 103 (2006) 2098-2102.

[9] Gov N., Diffusion in Curved Fluid Membranes, Phys. Rev. E 73 (2006) 041918 (6pp).

[10] Hänggi P., Talkner P., and Borkovec M., Reaction-Rate Theory: Fifty Years After Kramers, Rev. Mod. Phys. 62 (1990) 251-342.

[11] Jacobs M., Diffusion Processes, Springer, New York 1967.

[12] Kalinary P. and Percus J., Corrections to the Fick-Jacobs Equation, Phys. Rev. E 74 (2006) 041203 (6pp). 
[13] Kärger J. and Ruthven D., Diffusion in Zeolites and Other Microporous Solids, Wiley, New York 1992.

[14] Naji A. and Brown F., Diffusion on Ruffled Membrane Surfaces, J. Chem. Phys. 126 (2007) 235103; Reister E. and Seifert U., Lateral Diffusion of a Protein on a Fluctuating Membrane, Europhys. Lett. 71 (2005) 859-865.

[15] Ogawa N., Fujii K., and Kobushkin A., Quantum Mechanics in Riemannian Manifold, Prog. Theor. Phys. 83 (1990) 894-905; Ogawa N., Fujii K., Chepilko N. and Kobushkin A., Quantum Mechanics in Riemannian Manifold. II, Prog. Theor. Phys. 85 (1991) 1189-1201; Ogawa N., The Difference of Effective Hamiltonian in Two Methods in Quantum Mechanics on Submanifold, Progr. Theor. Phys. 87 (1992) 513-517.

[16] Ogawa N., Curvature Dependent Diffusion Flow on Surface with Thickness, Phys. Rev. E 81 (2010) 061113 (8pp).

[17] Ogawa N., Diffusion in a Curved Tube, Phys. Lett. A 377 (2013) 2465-2471.

[18] Reguera D. and Rubi J., Kinetic Equations for Diffusion in the Presence of Entropic Barriers, Phys. Rev. E 64 (2001) 061106 (8pp).

[19] Yanagida E., Existence of Stable Stationary Solutions of Scalar Reaction Diffusion Equations in Thin Tubular Domains, Appl. Anal. 36 (1990) 171-188; Raugel G., Dynamics of Partial Differential Equations on Thin Domains, Lecture Note in Mathematics 1609, Springer, Berlin 1995.

[20] Zwanzig R., Diffusion Past an Entropic Barrier, J. Phys. Chem. 96 (1992) 3926-3930.

Naohisa Ogawa

Hokkaido Institute of Technology

006-8585 Sapporo Japan

E-mail address: ogawanao@hus.ac.jp 